\title{
Adult Attachment Style and Marital Satisfaction - an exploratory study
}

\author{
Pankti Shah ${ }^{1}$, Sargam Bihari ${ }^{2}$, Chitra Munshi ${ }^{3}$ \\ ${ }^{1}$ Ex-resident, ${ }^{2}$ Assistant Professor, Department of Psychology, L.S Raheja College of Commerce and Arts \\ Corresponding author: Dr. Chitra Munshi \\ Email-chitram3@yahoo.co.in
}

\begin{abstract}
Background: Marital satisfaction is an important element for successful family life and personal growth. Various factors have significant effects on the marital satisfaction, like financial management, personality issues, equalitarian roles, religious orientation, sexual relationship etc. In recent times there has been a rise in divorce rates, couples living separately, couples dissatisfied with their marriage, etc. This could possibly be because of individuals having different attachment styles entering into a relationship. Basic proposition of attachment theory is that internal models of attachment (starting right from infancy) remain relatively stable across the life span.

Methods: We interviewed 50 consecutive consenting clinically stable patients with schizophrenia (BPRS score < 31) and depressive episode (HDRS score < 08) following up in psychiatry OPD. Montreal Cognitive Assessment (MoCA) was used to assess cognition, Snaith-Hamilton Pleasure Scale (SHAPS) for anhedonia and WHOQOL-BREF for the quality of life in participants.

Results: This study examined links between adult attachment style and marriage satisfaction in Indian adults. 24 participants (13 females and 11 males) aged 25-45 years (Mean $=35.35, \mathrm{SD}=6.01117$ ), completed the Revised Adult Attachment Style (RAAS) (Collins,1996) and ENRICH Marital Satisfaction Scale (EMS) (Fowers \& Olson, 1993). All the participants were heterosexual and married

Conclusion:Results indicated that participants having Secure attachment style were more satisfied with their marriage than participants having Insecure (Fearful, Preoccupied, Dismissive) attachment style.
\end{abstract}

Keywords: Adult attachment style, Marital satisfaction, Indian adult.

(Paper received $-2^{\text {nd }}$ January 2018 , Peer review completed $-6^{\text {th }}$ January 2018)

(Accepted $-10^{\text {th }}$ January 2018)

\section{INTRODUCTION}

According to John Bowlby "Attachment theory is a way of... explaining the many forms of emotional distress and personality disturbance, including anxiety, anger, depression and emotional detachment, to which unwilling separation and loss give rise".

A motivational system called Attachment Behaviour System was evolutionarily designed to regulate emotional bond proximity between infant and caregiver for protection and survival. However, there are two factors of attachment: infant's perception to availability of the caregiver and their experience with the caregiver. This relation with the caregiver serves as prototype for later social relations..

Three attachment styles identified in infants were Secure, Avoidant, and Ambivalent/Anxious- according to Mary Ainsworth. This categorization was used to understand attachment style in adults for the first time in the context of romantic relationship and was found that $56 \%$ of participants identified themselves as Secure, $25 \%$ as Avoidant, and $19 \%$ as Anxious [1]. 
Model of self and others was used to propose a four-category adult attachment model [2]. Secure individuals have positive perception of self and others while Fearful have negative perception of self and others. Dismissive individuals have positive perception of self and negative perception of others; while Preoccupied have negative perception of self and positive perception of others.

\section{Statement of Problem}

Many articles have emphasized the importance of secure attachments for functioning of optimum interpersonal relationship. Research has found quality of relationship varying with difference in attachment style. Earliest interactions of infants with their caregivers shape their relationship into secure or insecure, which later acts as prototype for future relationships. Hence, researchers were interested in understanding if there was a difference caused in the satisfaction in marriage because of attachment style. Thus, this study was focused on the attachment style of Indian adults and their marital satisfaction.

A study by Kenneth N. Levy and Kristen M. Kelly focused on how people with different attachment style would get affected because of their partner's emotional or sexual infidelity. The results showed that dismissive avoidant participants were more jealous when their partners indulged into sexual than emotional infidelity. Dismissive individuals are more independent, attempt to minimize or constrict emotional experience, prefer autonomy and are sexually promiscuous than individuals who have other attachment styles. Participants having secure attachment style, including secure men, reported more jealousy regarding emotional than sexual infidelity.

It has been implied that sexual jealousy in males is one of the major causes of spouse battering and homicide in many cultures, as shown in a study conducted by Daly and Wilson and thus understanding the dynamics of sexual jealousy is important. An attachment perspective emphasises that the understanding of jealousy primarily depends on the quality of internal working models of past and current interpersonal relationships, thus suggesting that promoting secure attachment relationships reduces and prevents sexual jealousy in both the genders.

\section{Hypothesis}

\section{METHODOLOGY}

There is a difference in marital satisfaction between people with secure attachment style and insecure attachment style (Fearful, Preoccupied and Dismissive styles).

\section{Independent variable}

Attachment style of the participants, as scored on the 3 dimensions of Close, Depend, and Anxiety i.e. raw scores on these subscales were used to categorize participants into 4 categories of attachment styles (Scoring protocol mentioned in tools). Hence, participants showing Secure attachment were taken into Secure attachment style and participants showing Fearful, Dismissive and Preoccupied styles were taken into Insecure attachment style.

\section{Dependent variable}

Marital satisfaction of the participants, as scored on the EMS Scale, i.e. higher EMS score denoted higher marital satisfaction and lower EMS score denoted lower marital satisfaction.

\section{Procedure}

The questionnaire was made using Google Docs. Questionnaires were sent electronically (via link) to the participants. Participants were asked basic family details like number of years they had been married and number of kids they had. The researchers kept the survey anonymous so as to ensure, more or less, honest answers from participants, rather than manipulative ones.

\section{Sample size and sampling}

This study was carried out on a sample size of 24 people who were married. The sample consisted of 13 females $(54.16 \%)$ and 11 males $(45.83 \%)$. The design of this study was a randomized design and sampling procedure used was convenient sampling. The age group of the sample was between 25 and 45 . There were no inclusion and exclusion criteria followed as such; however the questionnaire was distributed in English language, hence people who didn't have good command on language were excluded. 


\section{Tools}

The questionnaire consisted of 2 scales: Revised Adult Attachment Scale and ENRICH Marital Satisfaction Scale [3].

Revised Adult Attachment Scale (RAAS) is an 18-item self report scale that asks participants to rate the extent to which they agree with the statements using 5-point Likert scale (where, 1- not at all characteristic of me, 5 - very characteristic of me). Collins's RAAS is a modified version of Adult Attachment scale developed by Collins and Read (1990). This scale consists of three dimensions; Close, Depend and Anxiety, which are used to identify the 4 attachment styles given by Bartholomew and Horowitz [4], viz. Secure, Fearful, Preoccupied and Dismissive). The Close dimension refers to the extent to which the person is comfortable with closeness and intimacy. The Depend dimension refers to the extent to which the person feels they can depend on others to be available when needed. The Anxiety dimension refers to the extent to which the person is worried about being rejected or unloved.

A scoring protocol was used in which individual's raw score in each sub scales was compared to theoretical mean cut off score of 3 on that dimension to categorize each individual into one of the four attachment styles. The scores of subscales Close and Depend were combined to form Close Dep.

Hence, (a) Secure style should receive score higher than the cut-off in Close Dep subscales and lower than the cut-off in Anxiety subscale. (b) Fearful style should receive score lower than the cutoff in CloseDep subscale and higher than the cut-off in Anxiety subscale. (c) Preoccupied style should receive higher than the cut-off in both Close Dep and Anxiety subscale. (d) Dismissive should receive score lower than the cutoff in both Close Dep and Anxiety subscale.

Accordingly, the participants were segregated into different attachment styles. Cronbach alpha coefficient was found to be .82 (Close), .80 (Depend), .83 (Anxiety).

ENRICH (evaluation and nurturing relationship issues, communication and happiness) Marital Satisfaction (EMS) Scale [3] is a 15- item self report scale that asks participants to rate the extent to which they agree with the statements using 5- point Likert scale

(1- strongly disagree, 5- strongly agree). EMS Scale is a shortened version of ENRICH Inventory (125 items), which consists of 12 domains, viz. Idealistic distortion, Marital Satisfaction, Personality Issues, Communication, Conflict Resolution, Financial Management, Leisure Activities, Sexual Relationship, Children and Parenting, Family and Friends, Equalitarian Roles, and Religious Orientation. The EMS Scale comprises of 2 subscales of Idealistic Distortion (ID) (5 items) and Marital Satisfaction (MS) scale (10 items). Out of these $10 \mathrm{MS}$ items, each item is used to measure each domain used in ENRICH Inventory. The 5 items of ID measures Marital conventionalization which is the tendency to show their marital relationship in positive light. Hence, EMS score is obtained by revising the Marital Satisfaction scores to correct for Idealistic

\section{Distortion using the formula}

EMS score $=$ PCT $-[(.40 \times$ PCT $)(.01 \times$ ID $)]$

Where, PCT - percentile score for individual MS scale

ID - percentile score for individual ID scale

Cronbach's alpha for internal reliability was .86. Reliability coefficient, for test-retest reliability assessed on 115 participants over 4 weeks, was found to be .86 . Concurrent validity of .73 was found with LockeWallace Marital Adjustment Test. Norms were developed on the basis of national sample of 2,112 couples.

\section{RESULTS}

EMS score of Secure individuals was taken as sample A and that of Insecure individuals was taken as sample B. The sample size was insufficient since there were only 20 participants (excluding 4 participants who didn't fall in any attachment style) hence non-parametric test was conducted using Mann-Whitney U Test. 
Mean ranks for

\begin{tabular}{|c|c|}
\hline $\begin{array}{c}\text { Sample A (Marital satisfaction score for Secure } \\
\text { attachment style) }\end{array}$ & $\begin{array}{c}\text { Sample B (Marital satisfaction score for insecure } \\
\text { attachment style including Fearful, Dismissive, } \\
\text { and Preoccupied) }\end{array}$ \\
\hline 13 & 8.1 \\
\hline
\end{tabular}

\begin{tabular}{|c|c|}
\hline $\mathrm{UA}=25.5$ & \multirow{2}{*}{$\mathrm{P}=0.0351$} \\
$\mathrm{z}=1.81$ & (one tailed test) \\
\hline
\end{tabular}

The results were found to be significant at 0.05 level for one-tailed test. Hence, null hypothesis was rejected and alternative directional hypothesis was accepted. Therefore, people with Secure attachment style were found to be more satisfied in their marriage than people with insecure attachment style (Fearful, Preoccupied and Dismissive styles).

Mean EMS Score Of Participants $(\mathrm{N}=20)$

\begin{tabular}{|c|c|c|c|c|c|}
\hline & \multirow{2}{*}{ Secure } & \multirow{2}{*}{ Insecure } & \multicolumn{3}{|c|}{ Insecure } \\
\cline { 4 - 6 } & & & Fearful & Preoccupied & Dismissive \\
\hline Total & 541.02 & 414.64 & 90.2 & 100.168 & 224.272 \\
\hline Mean & 51.402 & 41.464 & 45.1 & 33.389 & 44.854 \\
\hline $\begin{array}{c}\text { Standard } \\
\text { Deviation }\end{array}$ & 10.7079 & 13.360 & 20.22 & 2.460 & 15.049 \\
\hline
\end{tabular}

\section{DISCUSSION}

The aim of the study was to examine links between married individuals and their attachment style and how the attachment style had affected their marital satisfaction. The prime focus was on the association between adult attachment which included attachment dimensions (Close, Depend, and Anxiety), as well as attachment styles (Secure, Fearful, Dismissive and Preoccupied) in marriage life.

Attachment is defined as: "An affectional tie that one person (or animal) forms to another specific individual" (Ainsworth, 1969). "Attachment refers to the relationship... the affective bond between infant and caregiver".

The study was conducted on 24 participants out of which 13 were females and 11 were males. However, the scores of four participants ( 3 females and 1 male) fell on the midpoint and thus couldn't be allotted to any of the attachment styles. Therefore, they were discarded, leaving sample size to 20 .

Five of each, female and male participants were married for anywhere ranging between 1-11 years, and the remaining 5 from each category were married for 9-20+ years.

On reviewing Table 1, it was found that out of the total sample size, 5 males and 5 females were found Secure and 5 males and 5 females were found Insecure. Out of the Insecure population, (a) Fearful - 1 male, 1 female; (b) preoccupied - 2 males, 1 female; (c) Dismissive - 2 males, 3 females.

On reviewing Table 2, it was seen that mean EMS score of participants with Secure attachment style was 51.402, which was also the highest mean EMS score obtained by any attachment style. This is because 
people with Secure attachment style tend to have a positive view of self and others [4]. They see their relationship in a positive light. They are able to trust others and are comfortable with close and intimate relationship [4]. They are capable of handling interpersonal difficulties with ease and are open to conversations. These characteristics make them the healthiest of 4 attachment styles, allowing them to be most satisfied with their marriage [5-7].

On reviewing Table 2, it was seen that mean EMS score of participants with Preoccupied attachment style was 33.389, which was the lowest mean EMS score. Preoccupied people have negative view of self and positive view of others [4]; hence they are always anxious about their partner leaving them. There is a constant need for validation from partner, which the partner might not always be able to fulfil, leading to less marital satisfaction; this constant need of their partner forms a codependence which is dysfunctional because neither of the partner matures further. People with serious relationship issues have reported cold, distant, neglectful caregiver [5].

It was also seen that the mean EMS score of participants with Dismissive was found to be 44.854 . Dismissive individuals hold positive perceptions about themselves and negative perception about others [4]. They are avoidant in nature, hence they are independent, prefer autonomy, are self sufficient and selfreliant and have their self esteem intact [4]. Therefore, people with Dismissive style tend to have highest satisfaction score in Insecure attachment styles, since they don't reply on their partners to be happy. However, because they have negative view of others their avoidant nature is an act of defence on denying intimacy which makes it an insecure attachment style [4].

The mean EMS score of participants with Fearful was found to be 45.1. Individuals with Fearful style hold negative perception about themselves and others [4,5]. They are avoidant in nature, making them uncomfortable in close and intimate relationships; they show social insecurity and lack of assertiveness and are most likely to report interpersonal problems [4]. Hence, people with fearful type are least satisfied with their marriage. However, the researchers found mean EMS score of Fearful to be highest in Insecure attachment style. This could be because of inadequate sample size ( $\mathrm{N}=2$ for Fearful).

\section{Methodological Error}

1. The sample size consisted of 24 participants, out of which 4 participants were excluded because they didn't fall under any category of attachment style (as their scores fell on midpoint). Hence, there were only 20 participants as sample size, which is relatively very small.

2. Mean EMS score of participants with Fearful attachment style was found to be highest in Insecure attachment style. This could be because of inadequate sampling i.e. more participants are required who are ideal representatives of the attachment style.

3. Since the questionnaire was sent via link, some people were not able to open the link, probably because it didn't support their cell phones.

\section{Confounding variables}

1. People in India prefer to keep their marriage life secret which could act as a possible confounding variable. Though the survey was kept anonymous, people might have not filled the form honestly which could have affected the results of the survey.

2. Although the researchers distributed the questionnaire to individuals who had good command on english language, it was possible that there could be misunderstanding ofcertain questions.

\section{REFERENCES}

1. Hazan C, Shaver P. Romantic love conceptualized as an attachment process. J Personal Soc Psychol 1987;52(3):511-8.

2. Bartholomew K, Horowitz LM. Attachment styles among young adults: a test of a four-category model. J Personal Soc Psychol 1991;61(2):226-32.

3. Fowers BJ, Olson DH. ENRICH Marital Satisfaction Scale: A brief research and clinical tool. J Fam Psychol 1993;7(2):176-88.

4. Griffin DW, Bartholomew K. Models of the self and other: Fundamental dimensions underlying measures of adult attachment. J Personal Soc Psychol 1994;67(3):430-8. 
5. Adamczyk K, Bookwala J. Adult attachment and single vs. partnered relationship status in Polish university students. Psychological Topics 2013;22(3):481-90.

6. Fitton V, Mooradian J. Attachment: Indicators from Caregivers and Toddlers-Implications for. Professional Development: The International Journal of Continuing. 2008;11(2):36-44.

7. Levy KN, Kelly KM. Sex differences in jealousy: A contribution from attachment theory. Psychol Sci 2010;21(2):168-73.

$* * * * * * * * * * * * * * * * * * * * * * *$

Acknowledgements - Nil

Conflict of Interest - Nil

Funding - Nil 